\title{
The Mitochondrial Tricarboxylate Carrier of Silver Eel: Chemical Modification by Sulfhydryl Reagents
}

\author{
Loredana Capobianco, Tecla Impagnatiello, Alessandra Ferramosca and Vincenzo Zara* \\ Dipartimento di Scienze e Tecnologie Biologiche ed Ambientali, Università di Lecce, Via Prov.le Lecce-Monteroni, I-73100 Lecce, Italy
}

Received 10 October 2003, Accepted 16 December 2003

\begin{abstract}
The tricarboxylate (or citrate) carrier was purified from eel liver mitochondria and functionally reconstituted into liposomes. Incubation of the proteoliposomes with various sulfhydryl reagents led to inhibition of the reconstituted citrate transport activity. Preincubation of the proteoliposomes with reversible $\mathrm{SH}$ reagents, such as mercurials and methanethiosulfonates, protected the eel liver tricarboxylate carrier against inactivation by the irreversible reagent $\mathbf{N}$-(1-pyrenyl)maleimide (PM). Citrate and L-malate, two substrates of the tricarboxylate carrier, protected the protein against inactivation by sulfhydryl reagents and decreased the fluorescent PM bound to the purified protein. These results suggest that the eel liver tricarboxylate carrier requires a single population of free cysteine(s) in order to manifest catalytic activity. The reactive cysteine(s) is most probably located at or near the substrate binding site of the carrier protein.
\end{abstract}

Keywords: Mitochondria, Reactive cysteine(s), Silver eel, Sulfhydryl reagents, Tricarboxylate carrier

\section{Introduction}

Mitochondria contain several integral proteins in the inner membrane which are responsible for the transport of charged metabolites across the lipid bilayer. These proteins exhibit similar structural properties and are therefore grouped in the

Abbreviations: 1,2,3-BTA, 1,2,3-benzenetricarboxylic acid; DTE, dithioerythritol; MTSEA, (2-aminoethyl)methanethiosulfonate hydrobromide; MTSES, sodium (2-sulfonatoethyl)methanethiosulfonate; NEM, N-ethylmaleimide; PM, N-(1-pyrenyl)maleimide; p-OHMB, p(hydroxymercuri)benzoic acid; SDS, sodium dodecyl sulfate; SDSPAGE, sodium dodecyl sulfate-polyacrylamide gel electrophoresis.

*To whom correspondence should be addressed.

Tel/Fax: +39-0832-298705

E-mail: vincenzo.zara@unile.it family of mitochondrial metabolite carriers (Palmieri, 1994; Kaplan, 2001; Zara et al., 2003). The tricarboxylate (or citrate) carrier belongs to this family and catalyzes the transport of citrate in exchange for a tricarboxylate, a dicarboxylate (L-malate) or phosphoenolpyruvate (Robinson et al., 1971; Palmieri et al., 1972). This carrier protein plays a central role in intermediary metabolism because it supplies the cytosol with acetyl units (deriving from the transported citrate) necessary for the de novo fatty acid and cholesterol biosyntheses (Meijer and van Dam, 1974; Conover, 1987). The tricarboxylate carrier has been investigated in detail mainly in mammals and in the yeast Saccharomyces cerevisiae. In fact, this transporter has been purified from liver mitochondria of rat (Bisaccia et al., 1989; Kaplan et al., 1990) and beef (Claeys and Azzi, 1989) and kinetically characterized after reconstitution into liposomes (Bisaccia et al., 1990). The rat liver tricarboxylate carrier has been cloned (Kaplan et al., 1993) and then overexpressed in bacteria (Xu et al., 1995). This transport protein has also been characterized in yeast, both structurally and functionally (Kaplan et al., 1995, 2000a, 2000b; Kotaria et al., 1999, Xu et al., 2000).

Recently, the tricarboxylate carrier has been purified to homogeneity from liver mitochondria of yellow and silver eels and functionally reconstituted into liposomes (Zara et al., 1996, 2000). The kinetic characterization of eel liver tricarboxylate carrier, carried out in proteoliposomes, revealed interesting differences with respect to the functional properties exhibited by the homologous protein from rat liver mitochondria (Zara et al., 1998). Briefly, under optimal conditions, the eel liver tricarboxylate carrier showed $K_{m}$ and $V_{\max }$ values for the uptake of citrate in reconstituted liposomes which were significantly different (about half and five times the previous values, respectively) from those measured with the same carrier from rat liver. In addition, unlike previous results obtained with the mammalian tricarboxylate carrier, phosphatidylethanolamine increased and phosphatidylinositol and phosphatidylserine decreased the activity of the eel liver tricarboxylate carrier. Very recently, the transmembrane 
organization and the oligomeric state of this transport protein has been investigated in eel liver mitochondria (Capobianco et al., 2002).

The investigation of functional groups and of their role in substrate transport is a useful way to obtain information about the molecular mechanism of a carrier system across the lipid bilayer of a biological membrane. In this study we report the interaction of a different set of sulfhydryl reagents with the purified tricarboxylate carrier of eel liver mitochondria reconstituted into liposomes. All the various SH reagents tested inhibited the citrate transport activity, even though with varying degrees of efficiency. Interestingly, the eel liver tricarboxylate carrier showed only one population of $\mathrm{SH}$ group(s) which was able to interact with the cysteine reagents. Citrate and L-malate protected the reconstituted carrier protein against the sulfhydryl reagents-mediated inhibition. This study is the first investigation into the functional groups of a metabolite carrier protein of fish mitochondria.

\section{Materials and Methods}

Chemicals Amberlite XAD-2, Sephadex G-75, mersalyl, p(hydroxymercuri)benzoic acid (p-OHMB), Pipes, cardiolipin, Triton X-100, Triton X-114, SDS, 1,2,3-benzenetricarboxylic acid (1,2,3BTA), dithioerythritol (DTE), N-ethylmaleimide (NEM) and N-(1pyrenyl)maleimide (PM) were obtained from Sigma (St. Louis, USA); egg yolk phospholipids (phosphatidylcholine from eggs) from Fluka (Buchs, Switzerland); (2-aminoethyl)methanethiosulfonate hydrobromide (MTSEA) and sodium (2-sulfonatoethyl)methanethiosulfonate (MTSES) from Fluorescent Dyes Inc.; hydroxyapatite (Bio-Gel HTP) from Bio-Rad (Hercules, USA); Matrex Gel Blue B from Amicon (Billerica, USA); $\left[1,5-{ }^{14} \mathrm{C}\right]$ citrate from Amersham (Piscataway, USA). All other reagents were of analytical grade.

Animals European silver eels (Anguilla anguilla) (about $200 \mathrm{~g} /$ each) were kept in sea-water aquaria and treated as previously described in (Zara et al., 2000) before being sacrificed.

Purification of the mitochondrial tricarboxylate carrier Liver mitochondria were obtained from silver eels as previously described (De Pinto et al., 1991). The isolated eel liver mitochondria were divided in aliquots of $12 \mathrm{mg}$ protein/each, frozen and stored at $80^{\circ} \mathrm{C}$. The purification of the tricarboxylate carrier from liver mitochondria of silver eels was carried out by sequential chromatography of the Triton X-100 mitochondrial extract on hydroxyapatite and Matrex Gel Blue B, essentially as previously described (Zara et al., 1996, 2000). Briefly, eel liver mitochondria were solubilized with $3 \%$ Triton X-100, $20 \mathrm{mM}$ $\mathrm{Na}_{2} \mathrm{SO}_{4}, 1 \mathrm{mM}$ EDTA and $10 \mathrm{mM}$ Pipes (pH 7.0) at a final concentration of $12 \mathrm{mg}$ protein $/ \mathrm{ml}$. After $10 \mathrm{~min}$ at $0^{\circ} \mathrm{C}$, the solubilized mitochondria were centrifuged at $25,000 \times g$ for $20 \mathrm{~min}$ at $2-4^{\circ} \mathrm{C}$. The obtained supernatant (referred to as mitochondrial extract) was supplemented with $2 \mathrm{mg} / \mathrm{ml}$ cardiolipin and chromatographed onto dry hydroxyapatite columns $(600 \mu \mathrm{l}$ supernatant onto $600 \mathrm{mg}$ packed hydroxyapatite). The elution was performed at $2^{\circ} \mathrm{C}$ with a buffer containing $0.5 \%$ Triton X-100 and 5
$\mathrm{mM}$ citrate $(\mathrm{pH}$ 7.0). The first $600-700 \mu \mathrm{l}$ of the eluate were collected. The hydroxyapatite eluate was then applied to a column containing $1.2 \mathrm{ml}$ of Matrex Gel Blue B. Contaminating proteins were eluted from this column first with $1 \mathrm{ml}$ of washing buffer consisting of $0.1 \%$ Triton X-114, $50 \mathrm{mM} \mathrm{NaCl}, 20 \mathrm{mM}$ Hepes, $1 \mathrm{mM}$ EDTA (pH 7.2), then with $4 \mathrm{ml}$ of washing buffer supplemented with $100 \mathrm{mM}$ citrate, followed by $1 \mathrm{ml}$ of the same buffer plus $5 \mathrm{mM}$ citrate. The eel liver tricarboxylate carrier was specifically eluted from the Matrex Gel Blue B with the addition of $1 \mathrm{ml}$ of a buffer containing $1 \%$ Triton X-114, $50 \mathrm{mM} \mathrm{NaCl}, 20 \mathrm{mM}$ Hepes, $1 \mathrm{mM}$ EDTA, $5 \mathrm{mM}$ citrate (pH 7.2), and $5 \mathrm{mg} / \mathrm{ml}$ cardiolipin.

Reconstitution of the purified protein into liposomes and transport measurements The purified tricarboxylate carrier was reconstituted into liposomes by removing the detergent with the use of Amberlite XAD-2 (Palmieri et al., 1995). The mixture chromatographed onto this resin contained: $40 \mu \mathrm{l}$ of the eluate from Matrex Gel Blue B (containing the tricarboxylate carrier purified to homogeneity), $90 \mu \mathrm{l}$ of $10 \%$ Triton X-114, $20 \mu \mathrm{l}$ of $20 \mathrm{mg} / \mathrm{ml}$ cardiolipin, $90 \mu \mathrm{l}$ of $10 \%$ egg yolk phospholipids in the form of sonicated liposomes, $70 \mu \mathrm{l}$ of $100 \mathrm{mM}$ Pipes (pH 7.0) and $35 \mu \mathrm{l}$ of $200 \mathrm{mM}$ citrate in a final volume of $700 \mu \mathrm{l}$. This mixture was passed ten times through the same Amberlite XAD-2 column as previously described (Zara et al., 1998), thereby obtaining the proteoliposomes. After the external substrate had been removed by chromatography onto Sephadex G-75 (Zara et al., 1996, 1998), the proteoliposomes were distributed in reaction vessels $(150 \mu \mathrm{l} / \mathrm{each})$. The preincubation of the vesicles with different sulfhydryl reagents in the presence and in the absence of substrates was performed as described in the legend to the figures. Transport, carried out at $25^{\circ} \mathrm{C}$, was started by the addition of the indicated concentrations of $\left[1,5-{ }^{14} \mathrm{C}\right]$ citrate to proteoliposomes and stopped, after the desired time, by adding $20 \mathrm{mM}$ of the specific inhibitor 1,2,3-BTA. In control samples, 1,2,3-BTA was added together with the labeled substrate. After the transport measurements, the external radioactivity was removed on Sephadex G-75, and the internal one was determined by scintillation counting. The reconstituted tricarboxylate transport activity was then calculated by subtracting the control from the experimental values.

Labeling of the reconstituted tricarboxylate carrier with PM The eel liver tricarboxylate carrier was labeled in proteoliposomes with $1 \mathrm{mM} \mathrm{PM}$ for $30 \mathrm{~min}$ at $25^{\circ} \mathrm{C}$, in the presence and in the absence of the protein substrate, essentially as described in (Capobianco et al., 1996). The SH reagent not bound to the reconstituted protein was removed by passing the proteoliposomes through a Sephadex G-75 column. The eluate obtained from this column was precipitated with a 20 -fold excess of cold acetone for $4 \mathrm{~h}$ at $-20^{\circ} \mathrm{C}$ and then centrifuged at $45,000 \times g$ for $10 \mathrm{~min}$ at $2^{\circ} \mathrm{C}$. The pellet was delipidated as described in (Wessel and Flügge, 1984) with the modifications reported in (Capobianco et al., 1996). The precipitated and delipidated protein was subsequently analyzed by standard SDS-PAGE (Laemmli, 1970) and either stained with Coomassie Brilliant Blue R-250 or analyzed by fluorography as described in (Zara and Palmieri, 1988).

Titration of SH groups The free sulfhydryl groups of eel liver tricarboxylate carrier were titrated with $\mathrm{p}-\mathrm{OHMB}$ essentially as 

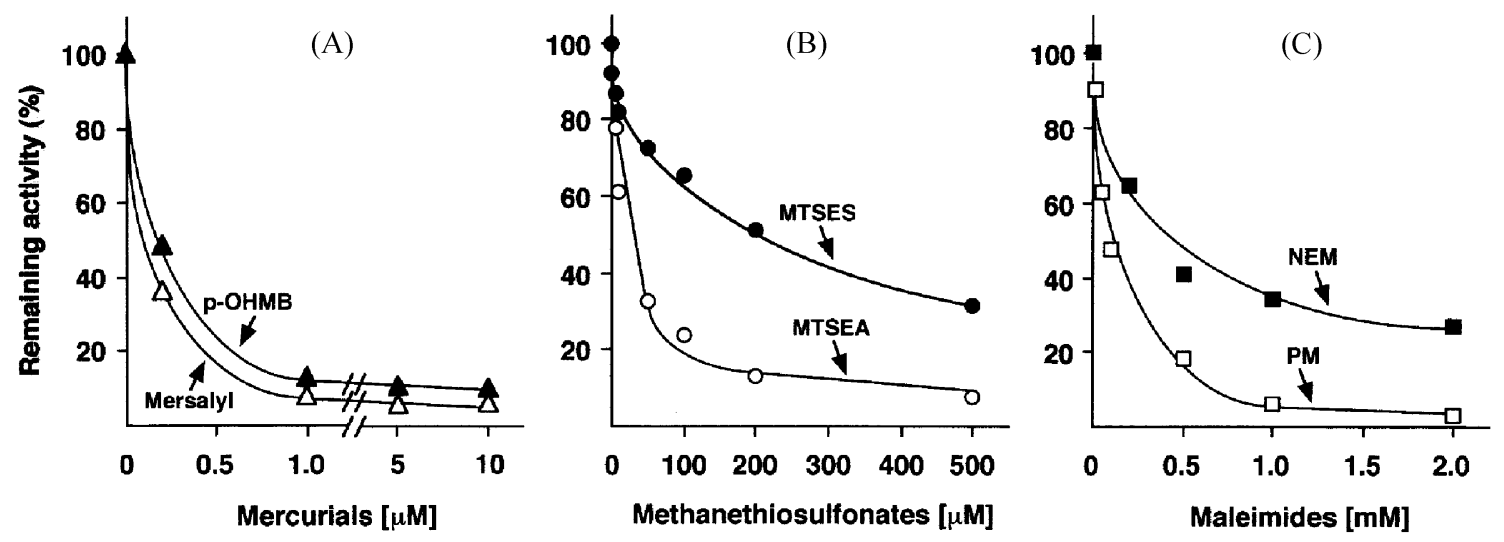

Fig. 1. Inhibition of the reconstituted tricarboxylate carrier by mercurials (A), methanethiosulfonates (B) and maleimides (C). Proteoliposomes were incubated with the indicated concentrations of Mersalyl (open triangle), p-OHMB (solid triangle), MTSES (solid circle), MTSEA (open circle) and NEM (solid square) for $5 \mathrm{~min}$ at $25^{\circ} \mathrm{C}$, or with PM (open square) for $30 \mathrm{~min}$ at $25^{\circ} \mathrm{C}$. Citrate transport activity was then measured by adding $50 \mu \mathrm{M}\left[{ }^{14} \mathrm{C}\right]$ citrate and stopped after $1 \mathrm{~min}$, as described in Materials and methods. The percentage of remaining activity with respect to the control is reported. The control activity in the absence of mercurials (A) was $5345 \mathrm{nmol} \times \mathrm{min}^{-1} \times \mathrm{mg}_{\text {protein }}{ }^{-1}$, in the absence of methanethiosulfonates (B) was $5,245 \mathrm{nmol} \times \mathrm{min}^{-1} \times \mathrm{mg}_{\text {protein }}^{-1}$, and in the absence of maleimides (C) was $5,105 \mathrm{nmol} \times \min ^{-1} \times \mathrm{mg}_{\text {protein }}{ }^{-1}$.

described in (Boyer, 1954). Briefly, the purified tricarboxylate carrier was incubated with $1 \mu \mathrm{M}$ p-OHMB in $50 \mathrm{mM}$ phosphate buffer, $\mathrm{pH} 7.0$, at $25^{\circ} \mathrm{C}$. The reaction of the protein with $\mathrm{p}-\mathrm{OHMB}$ formed a mercaptide that was monitored by measuring the absorbance increase at $255 \mathrm{~nm}$.

Other Methods Protein concentration was determined using the Bradford method (Bradford, 1976), the Lowry method modified for the presence of Triton (Dulley and Grieve, 1975), and as previously reported in (Fiermonte et al., 1998).

\section{Results}

Inhibition of the reconstituted tricarboxylate carrier activity by various sulfhydryl reagents The effect of different sulfhydryl reagents, including mercurials, methanethiosulfonates and maleimides, on the purified and reconstituted eel liver tricarboxylate carrier is reported in Fig. 1. This figure shows that the preincubation of the proteoliposomes with p-OHMB and mersalyl (Fig. 1A), with MTSES and MTSEA (Fig. 1B), and with NEM and PM (Fig. 1C), caused a concentrationdependent inhibition of the reconstituted citrate transport activity. In these experiments the concentration of the sulfhydryl reagents was varied as indicated in the figure in the absence of external citrate. With all the reagents tested, the inhibition of the transport activity was dependent on the incubation time of the proteoliposomes with the sulfhydryl reagents (data not shown). PM was able to inhibit the reconstituted citrate carrier when the proteoliposomes were incubated with this reagent for $30 \mathrm{~min}$ at $25^{\circ} \mathrm{C}$. On the contrary, using NEM, methanethiosulfonates and mercurials, the incubation time required to inhibit the reconstituted citrate transport activity was shortened to $5 \mathrm{~min}$ at $25^{\circ} \mathrm{C}$. Figure 1 shows that all these $\mathrm{SH}$ specific reagents inhibited the tricarboxylate transport activity, even though with different degrees of potency. p-OHMB and mersalyl showed the highest reactivity. The half-maximal inhibition $\left(\mathrm{IC}_{50}\right)$ of the reconstituted tricarboxylate carrier activity was found to be $0.15 \mu \mathrm{M}$ and $0.2 \mu \mathrm{M}$ for mersalyl and p-OHMB, respectively. Between the two methanethiosulfonates, the most effective inhibitor was MTSEA with an $\mathrm{IC}_{50}$ of $35 \mu \mathrm{M}$, while MTSES had an $\mathrm{IC}_{50}$ of $200 \mu \mathrm{M}$. Among the sulfhydryl reagents tested, NEM was the least powerful inhibitor. In fact, PM and NEM exhibited an $\mathrm{IC}_{50}$ of $0.1 \mathrm{mM}$ and $0.5 \mathrm{mM}$, respectively. It is clear from the data reported in Fig. 1 that the sulfhydryl reagents tested, with the exception of MTSES and NEM, almost completely inhibited the reconstituted tricarboxylate carrier activity. In order to check whether infinite concentrations of MTSES and NEM would cause 100\% inhibition, reciprocal plots were made to estimate the maximum inhibition at infinite inhibitor concentrations. Maximum inhibitions of $70.4 \%$ and $86.2 \%$ were obtained for MTSES and NEM, respectively (data not shown). Since all the reagents used in this study are known to react specifically with cysteines, these results show that the eel liver tricarboxylate carrier possesses at least one functionally important sulfhydryl group.

Titration of cysteine residues Several unsuccessful attempts were made to titrate the $\mathrm{SH}$ groups of the reconstituted protein which react with $\mathrm{p}-\mathrm{OHMB}$. These failed due to the high turbidity of the proteoliposomes during the spectrophotometric determination according to (Boyer, 1954) and to the very low amount of tricarboxylate carrier in the reconstituted system. A different approach, performed with the purified eel liver tricarboxylate carrier, gave 3.0 reactive sulfhydryl groups per protein monomer after an incubation of $5 \mathrm{~min}$ at $25^{\circ} \mathrm{C}$ with $1 \mu \mathrm{M}$ p-OHMB. The denaturation of the native and functional protein with $0.5 \%$ SDS gave 6.0 reactive $\mathrm{SH}$ groups per 
Table 1. Protection of the reconstituted tricarboxylate carrier by reversible SH reagents against PM inhibition

\begin{tabular}{|c|c|c|c|c|}
\hline \multicolumn{3}{|c|}{ Addition at } & \multirow{2}{*}{$\begin{array}{c}\text { Citrate uptake } \\
\left(\mathrm{nmol} \times \min ^{-1} \times \mathrm{mg} \text { proteins }{ }^{-1}\right)\end{array}$} & \multirow{2}{*}{$\begin{array}{c}\text { Remaining activity } \\
(\%)\end{array}$} \\
\hline 0 min & $5 \mathrm{~min}$ & $10 \mathrm{~min}$ & & \\
\hline- & - & - & 5250 & 100 \\
\hline- & - & DTE & 5190 & 100 \\
\hline MTSES & - & - & 1680 & 32 \\
\hline MTSES & - & DTE & 4827 & 93 \\
\hline p-OHMB & - & - & 105 & 2 \\
\hline p-OHMB & - & DTE & 4671 & 90 \\
\hline Mersalyl & - & - & 368 & 7 \\
\hline Mersalyl & - & DTE & 4412 & 85 \\
\hline- & PM & - & 1418 & 27 \\
\hline- & PM & DTE & 1038 & 20 \\
\hline- & NEM & - & 2100 & 40 \\
\hline- & NEM & DTE & 2336 & 45 \\
\hline MTSES & PM & DTE & 3633 & 70 \\
\hline p-OHMB & PM & DTE & 3425 & 66 \\
\hline PM & MTSES & DTE & 1038 & 20 \\
\hline
\end{tabular}

Proteoliposomes were incubated at $25^{\circ} \mathrm{C}$ with the sulfhydryl reagents reported below, and DTE was added at the time indicated. After incubation, citrate transport activity was measured by adding $50 \mu \mathrm{M}\left[{ }^{14} \mathrm{C}\right]$ citrate. The sulfhydryl reagents were used at the following concentrations: $0.5 \mathrm{mM}$ MTSES, $20 \mu \mathrm{M}$ p-OHMB, $5 \mu \mathrm{M}$ Mersalyl, $0.5 \mathrm{mM}$ PM, $1 \mathrm{mM}$ NEM, and $10 \mathrm{mM}$ DTE.

monomer, in perfect accordance with sequence data (Zara et al., manuscript in preparation) indicating that the eel liver tricarboxylate carrier possesses six cysteines.

Competition among the sulfhydryl reagents for the same cysteine residue(s) It is well known that the binding of mercurials and methanethiosulfonates to the cysteine residues of a protein is efficiently reversed by DTE, while the binding of maleimides is not, thereby rendering this last interaction irreversible. Taking advantage of this different reactivity, we have investigated whether the various reagents tested were able to bind to the same or to different SH group(s) of the purified eel liver tricarboxylate carrier.

Table 1 shows that after inactivation of the reconstituted tricarboxylate carrier by MTSES, p-OHMB and mersalyl, the transport activity could be reactivated, almost completely, by treatment with DTE. On the contrary, after treatment with PM and NEM, the addition of DTE did not restore the reconstituted transport activity. In further experiments, proteoliposomes were first incubated with MTSES and p-OHMB (reversible reagents) and then PM was added. The data given in Table 1 shows that a subsequent treatment of these proteoliposomes with DTE was able to restore the tricarboxylate transport activity significantly. On the contrary, no restoration of carrier activity was observed when the proteoliposomes were preincubated with PM, subsequently treated with MTSES, and finally with DTE. These results suggest that p-OHMB, MTSES and PM react mainly with the same cysteine residue(s) of the eel liver tricarboxylate carrier.
Protection of the reconstituted tricarboxylate carrier activity by substrates against inhibition by sulfhydryl reagents Specific protection against the effect of a particular inhibitor, determined by the presence of a protein substrate, is generally considered evidence for the interaction of the inhibitor at or near the substrate binding site. To investigate this aspect, we analyzed the effect of two substrates of the tricarboxylate carrier, citrate and L-malate, on the inhibition of the transport activity caused by the sulfhydryl reagents. Liposomes reconstituted with the purified tricarboxylate carrier were incubated with different $\mathrm{SH}$ reagents in the presence and in the absence of the two substrates. In these experiments, the concentration of the sulfhydryl reagents was kept sufficiently low to obtain only partial transport inhibition. The data reported in Fig. 2 show that the inhibition of citrate transport by $0.2 \mathrm{mM}$ MTSES or $0.04 \mathrm{mM}$ MTSEA (A) or 0.1 $\mathrm{mM}$ PM (B) was progressively abolished by the presence of increasing concentrations of citrate during the treatment of the proteoliposomes with these reagents. The same results (data not shown) were obtained in the presence of L-malate outside proteoliposomes. It is worth noting that the protective effect of citrate was specific, since other anions such as malonate (Fig. $2 \mathrm{~A}$ and $2 \mathrm{~B}$ ) or glutamate (data not shown), which are neither substrates nor inhibitors of the tricarboxylate carrier, exhibited no protection. Furthermore, under the conditions used in the experiments reported in Fig. 2A and 2B, significant protection was obtained with citrate concentrations close to the transport affinity $\left(K_{m}\right)$ of the reconstituted eel liver tricarboxylate carrier for this substrate (Zara et al., 1998). Moreover, these results support those obtained in the following experiment. 

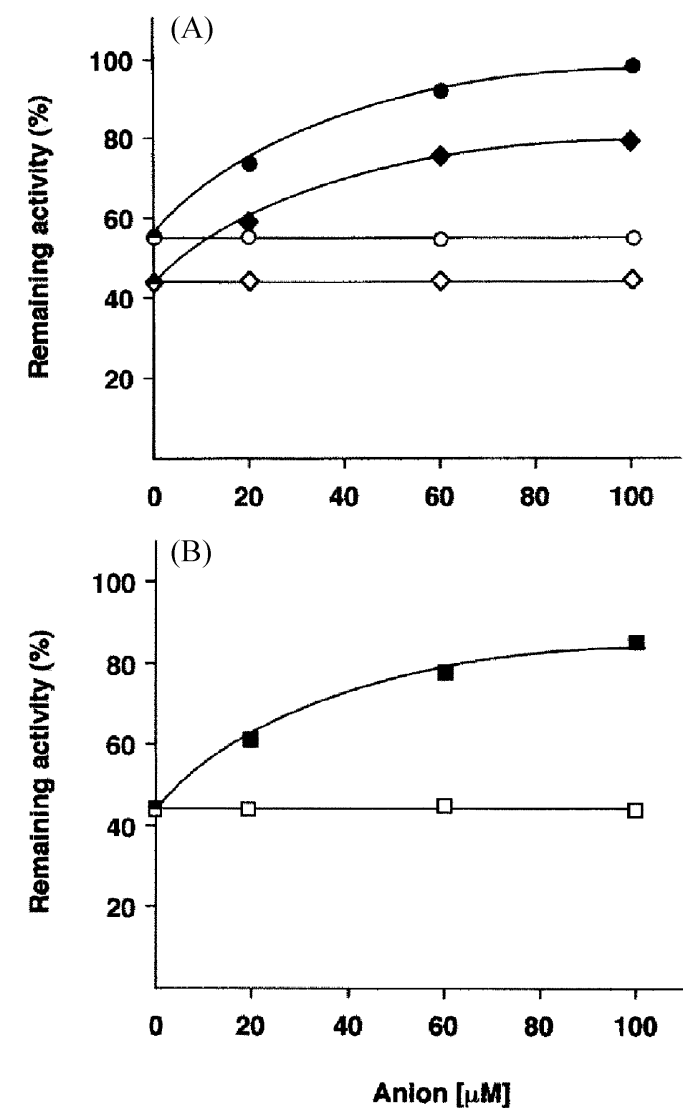

Fig. 2. Protection of the reconstituted citrate transport activity by substrates against inhibition caused by sulfhydryl reagents. (A) Proteoliposomes were incubated for $5 \mathrm{~min}$ at $25^{\circ} \mathrm{C}$ with $0.2 \mathrm{mM}$ MTSES (solid and open circle) or $0.04 \mathrm{mM}$ MTSEA (solid and open rhombus) and the indicated concentrations of citrate (solid circle and solid rhombus) or malonate (open circle and open rhombus). (B) Proteoliposomes were incubated for $30 \mathrm{~min}$ at $25^{\circ} \mathrm{C}$ with $0.1 \mathrm{mM}$ PM (solid and open square) and the indicated concentrations of citrate (solid square) or malonate (open square). Citrate transport activity was then measured by adding different concentrations of $\left[{ }^{14} \mathrm{C}\right]$ citrate to reach the same external concentration of $0.1 \mathrm{mM}$ in all the samples.

Figure 3 shows the SDS-PAGE and the fluorography of the eel liver tricarboxylate carrier recovered from proteoliposomes incubated with the fluorescent PM in the presence and in the absence of external citrate. The fluorescence associated with the tricarboxylate carrier (Fig. 3B, lane 1) was markedly diminished when the proteoliposomes were incubated with PM in the presence of $1 \mathrm{mM}$ citrate (Fig. 3B, lane 2). Taken together, these results suggest that the cysteine residue(s) is inaccessible to the sulfhydryl reagents when the substrate is bound to the carrier protein.

\section{Discussion}

The results obtained in this study indicate that the eel liver

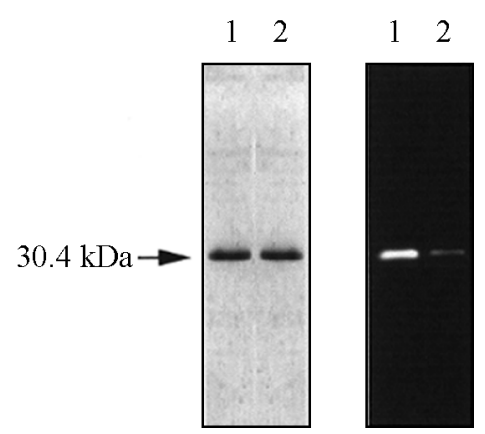

Fig. 3. Influence of citrate on the labeling of the reconstituted tricarboxylate carrier by PM. Proteoliposomes were preincubated for $30 \mathrm{~min}$ at $25^{\circ} \mathrm{C}$ with $1 \mathrm{mM} \mathrm{PM}$ (lanes 1 and 2) in the absence (lane 1) and in the presence (lane 2) of $1 \mathrm{mM}$ citrate. After removal of unbound PM, the carrier protein was delipidated and subjected to SDS-PAGE. (A) Coomassie Blue staining; (B) fluorography.

tricarboxylate carrier requires free cysteine(s) in order to catalyze the efflux of citrate from the mitochondrial matrix. Among the sulfhydryl reagents tested, the mercurials were the most powerful inhibitors. The higher reactivity of mercurials with respect to maleimides has also been reported in the case of the purified and reconstituted tricarboxylate carrier of mammalian mitochondria (Stipani and Palmieri, 1983; Bisaccia et al., 1989). In the latter case, however, higher concentrations of both reagents were required in order to obtain a degree of inhibition comparable to that found with the eel liver tricarboxylate carrier. For example, in the case of mercurials $0.2 \mathrm{mM}$ (Stipani and Palmieri, 1983) and $1 \mathrm{mM}$ (Bisaccia et al., 1989) of these reagents were required in order to get about $90 \%$ inhibition of the rat liver tricarboxylate transport activity, whereas in the case of maleimides 1-2 mM of NEM caused an inhibition of about $20-40 \%$ (Stipani and Palmieri, 1983; Bisaccia et al., 1989). It is also worth mentioning that a detailed study on the $\mathrm{SH}$ reagents inhibition and on the relative $\mathrm{IC}_{50}$ values has not yet been carried out for rat liver tricarboxylate carrier. The significance, at a molecular level, of the higher reactivity of mercurials is still unknown. However, the different inhibitory potency of these SH reagents can be explained, at least in part, by taking into account the microenvironment surrounding the reactive cysteine(s). In fact, the side group of the inhibitors can come into close contact with specific amino acid residues located in the vicinity of the reactive SH group(s) of the protein, thus influencing the inhibitor reactivity.

On the basis of the competition experiments previously reported, it is evident that some of the sulfhydryl reagents tested (i.e., p-OHMB, MTSES and PM) bind to the same SH group(s) of the tricarboxylate carrier. In fact, a significant recovery of activity (about 75\%) was observed, upon addition of DTE, in the samples treated with reversible reagents (MTSES and p-OHMB) before PM. However, the lack of 
complete recovery of citrate transport activity could be due to a small fraction of protein still able to interact with PM in the presence of reversible $\mathrm{SH}$ reagents.

The two substrates of the tricarboxylate carrier, citrate and Lmalate, were able to protect the transport activity against the inhibition caused by the sulfhydryl reagents. This means that the binding of the substrates renders the cysteine(s) inaccessible to the $\mathrm{SH}$ reagents. The most plausible explanation is that the reactive cysteine(s) of the tricarboxylate carrier is located at the substrate binding site or in close proximity to it. It cannot be excluded, however, that the substrate binding causes a conformational change in the tricarboxylate carrier, thereby decreasing the reactivity or accessibility of the cysteine residue(s) for $\mathrm{SH}$ reagents. According to this second hypothesis, the reactive cysteine(s) could be located in a protein region different from the substrate binding site.

On the basis of these and previous results obtained with the use of sulfhydryl reagents (for reviews, see Palmieri et al., 1996; Kaplan, 2001; Zara et al., 2003) it is clear that the mitochondrial metabolite carriers require free cysteines in order to function. A chemical modification of the $\mathrm{SH}$ groups strongly impairs the transport activity of the mitochondrial carriers. However, the role played by these residues inside the carrier proteins is still controversial. On one hand, on the basis of site-directed mutagenesis experiments (Palmieri et al., 1996; Xu et al., 2000; Hatanaka et al., 2001; Indiveri et al., 2002), it has been demonstrated that the cysteine residues do not play an essential role in the mechanism of translocation. It was therefore proposed that the transport inhibition caused by the $\mathrm{SH}$ reagents was a consequence of a steric effect and not due to the functional importance of the cysteine residues. On the other hand, a C73S mutant of the isoform 2 of the yeast ADP/ATP carrier (AAC) was inactive after reconstitution into liposomes, it showed a low level of bound cardiolipin and absolutely required this phospholipid for its function in the reconstituted system (Hoffmann et al., 1994). Very interestingly, it has recently been proposed that the conserved cysteine residues of the yeast AAC are important for the formation of a functional dimer of this protein in the inner mitochondrial membrane (Dyall et al., 2003). Finally, it has to be mentioned that the binding of mercurials to some metabolite carriers, such as the AAC (Dierks et al., 1990a), the aspartate/ glutamate carrier (Dierks et al., 1990a, 1990b), the carnitine carrier (Indiveri et al., 1992), the phosphate carrier (Stappen and Krämer, 1993) and the ornithine/citrulline carrier (Tonazzi and Indiveri, 2003) caused the transition from an antiport to a uniport transport mode.

Titration experiments of free $\mathrm{SH}$ groups carried out with the purified tricarboxylate carrier indicated that the protein monomer possesses three reactive cysteines. This finding does not necessarily mean that in the reconstituted system all three cysteines react with $\mathrm{p}-\mathrm{OHMB}$ in order to give the complete transport inhibition observed in Fig. 1A. In the proteoliposomal system some regions of the protein, and therefore some reactive cysteines, can be masked by the lipid bilayer which can thus reduce the accessibility of the protein to reagents. We have just determined the complete amino acid sequence of the eel liver tricarboxylate carrier (Zara et al., manuscript in preparation) and have discovered that this protein possesses six endogenous cysteines. We are therefore trying to overexpress the protein in E. coli and to reconstitute the purified protein in phospholipid vesicles. Furthermore, we intend to probe the role played by each of the six cysteine residues in the eel liver tricarboxylate carrier function by sitedirected mutagenesis experiments.

\section{References}

Bisaccia, F., De Palma, A. and Palmieri, F. (1989) Identification and purification of the tricarboxylate carrier from rat liver mitochondria. Biochim. Biophys. Acta 977, 171-176.

Bisaccia, F., De Palma, A., Prezioso, G. and Palmieri, F. (1990) Kinetic characterization of the reconstituted tricarboxylate carrier from rat liver mitochondria. Biochim. Biophys. Acta 1019, 250-256.

Boyer, P. D. (1954) Spectrophotometric study of the reaction of protein sulfhydryl groups with organic mercurials. J. Am. Chem. Soc. 76, 4331-4337.

Bradford, M. M. (1976) A rapid and sensitive method for the quantitation of microgram quantities of protein utilizing the principle of protein-dye binding. Anal. Biochem. 72, 248-254.

Capobianco, L., Bisaccia, F., Mazzeo, M. and Palmieri, F. (1996) The mitochondrial oxoglutarate carrier: sulfhydryl reagents bind to cysteine-184, and this interaction is enhanced by substrate binding. Biochemistry 35, 8974-8980.

Capobianco, L., Ferramosca, A. and Zara, V. (2002) The mitochondrial tricarboxylate carrier of silver eel: dimeric structure and cytosolic exposure of both $\mathrm{N}$ - and C-termini. $J$. Prot. Chem. 21, 515-521.

Claeys, D. and Azzi, A. (1989) Tricarboxylate carrier of bovine liver mitochondria. Purification and reconstitution. J. Biol. Chem. 264, 14627-14630.

Conover, T. E. (1987) Does citrate transport supply both acetyl groups and NADPH for cytoplasmic fatty acid synthesis? Trends Biochem. Sci. 12, 88-89.

De Pinto, V., Zara, V., Benz, R., Gnoni, G. V. and Palmieri, F. (1991) Characterization of pore-forming activity in liver mitochondria from Anguilla anguilla. Two porins in mitochondria? Biochim. Biophys. Acta 1061, 279-286.

Dierks, T., Salentin, A., Heberger, C. and Krämer, R. (1990a) The mitochondrial aspartate/glutamate and ADP/ATP carrier switch from obligate counterexchange to unidirectional transport after modification by SH-reagents. Biochim. Biophys. Acta 1028, 268-280.

Dierks, T., Salentin, A. and Krämer, R. (1990b) Pore-like and carrier-like properties of the mitochondrial aspartate/glutamate carrier after modification by SH-reagents: evidence for a preformed channel as a structural requirement of carriermediated transport. Biochim. Biophys. Acta 1028, 281-288.

Dulley, J. R. and Grieve, P. A. (1975) A simple technique for eliminating interference by detergents in the Lowry method of protein determination. Anal. Biochem. 64, 136-141.

Dyall, S., Agius, S. C., De Marcos Lousa, C., Trézéguet, V. and 
Tokatlidis, K. (2003) The dynamic dimerization of the yeast ADP/ATP carrier in the inner mitochondrial membrane is affected by conserved cysteine residues. J. Biol. Chem. 278, 26757-26764.

Fiermonte, G., Dolce, V. and Palmieri, F. (1998) Expression in Escherichia coli, functional characterization, and tissue distribution of isoforms $\mathrm{A}$ and $\mathrm{B}$ of the phosphate carrier from bovine mitochondria. J. Biol. Chem. 273, 22782-22787.

Hatanaka, T., Kihira, Y., Shinohara, Y., Majima, E. and Terada, H. (2001) Characterization of loops of the yeast mitochondrial ADP/ATP carrier facing the cytosol by site-directed mutagenesis. Biochem. Biophys. Res. Commun. 286, 936-942.

Hoffmann, B., Stöckl, A., Schlame, M., Beyer, K. and Klingenberg, M. (1994) The reconstituted ADP/ATP carrier activity has an absolute requirement for cardiolipin as shown in cysteine mutants. J. Biol. Chem. 269, 1940-1944.

Indiveri, C., Tonazzi, A., Dierks, T., Krämer, R. and Palmieri, F. (1992) The mitochondrial carnitine carrier: characterization of SH-groups relevant for its transport function. Biochim. Biophys. Acta 1140, 53-58.

Indiveri, C., Giangregorio, N., Iacobazzi, V. and Palmieri, F. (2002) Site-directed mutagenesis and chemical modification of the six native cysteine residues of the rat mitochondrial carnitine carrier: implications for the role of cysteine-136. Biochemistry 41, 8649-8656.

Kaplan, R. S., Mayor, J. A., Johnston, N. and Oliveira, D. L. (1990) Purification and characterization of the reconstitutively active tricarboxylate transporter from rat liver mitochondria. $J$. Biol. Chem. 265, 13379-13385.

Kaplan, R. S., Mayor, J. A. and Wood, D. O. (1993) The mitochondrial tricarboxylate transport protein. cDNA cloning, primary structure, and comparison with other mitochondrial transport proteins. J. Biol. Chem. 268, 13682-13690.

Kaplan, R. S., Mayor, J. A., Gremse, D. A. and Wood, D. O. (1995) High level expression and characterization of the mitochondrial citrate transport protein from the yeast Saccharomyces cerevisiae. J. Biol. Chem. 270, 4108-4114.

Kaplan, R. S., Mayor, J. A., Brauer, D., Kotaria, R., Walters, D. E. and Dean, A. M. (2000a) The yeast mitochondrial citrate transport protein. Probing the secondary structure of transmembrane domain IV and identification of residues that likely comprise a portion of the citrate translocation pathway. $J$. Biol. Chem. 275, 12009-12016.

Kaplan, R. S., Mayor, J. A., Kotaria, R., Walters, D. E. and Mchaourab, H. S. (2000b) The yeast mitochondrial citrate transport protein: determination of secondary structure and solvent accessibility of transmembrane domain IV using sitedirected spin labeling. Biochemistry 39, 9157-9163.

Kaplan, R. S. (2001) Structure and function of mitochondrial anion transport proteins. J. Membr. Biol. 179, 165-183.

Kotaria, R., Mayor, J. A., Walters, D. E. and Kaplan, R. S. (1999) Oligomeric state of wild-type and cysteine-less yeast mitochondrial citrate transport proteins. J. Bioenerg. Biomembr. 31, 543-549.

Laemmli, U. K. (1970) Cleavage of structural proteins during the assembly of the head of bacteriophage T4. Nature 227, 680685.

Meijer, A. J. and van Dam, K. (1974) The metabolic significance of anion transport in mitochondria. Biochim. Biophys. Acta 346, 213-244.
Palmieri, F., Stipani, I., Quagliariello, E. and Klingenberg, M. (1972) Kinetic study of the tricarboxylate carrier in rat-liver mitochondria. Eur. J. Biochem. 26, 587-594.

Palmieri, F. (1994) Mitochondrial carrier proteins. FEBS Lett. 346, 48-54.

Palmieri, F., Indiveri, C., Bisaccia, F. and Iacobazzi, V. (1995) Mitochondrial metabolite carrier proteins: purification, reconstitution, and transport studies. Methods Enzymol. 260, 349-369.

Palmieri, F., Bisaccia, F., Capobianco, L., Dolce, V., Fiermonte, G., Iacobazzi, V., Indiveri, C. and Palmieri, L. (1996) Mitochondrial metabolite transporters. Biochim. Biophys. Acta 1275, 127-132.

Robinson, B. H., Williams, G. R., Halperin, M. L. and Leznoff, C. C. (1971) Factors affecting the kinetics and equilibrium of exchange reactions of the citrate-transporting system of rat liver mitochondria. J. Biol. Chem. 246, 5280-5286.

Stappen, R. and Krämer, R. (1993) Functional properties of the reconstituted phosphate carrier from bovine heart mitochondria: evidence for asymmetric orientation and characterization of three different transport modes. Biochim. Biophys. Acta 1149, 40-48.

Stipani, I. and Palmieri, F. (1983) Purification of the active mitochondrial tricarboxylate carrier by hydroxylapatite chromatography. FEBS Lett. 161, 269-274.

Tonazzi, A. and Indiveri, C. (2003) Chemical modification of the mitochondrial ornithine/citrulline carrier by SH reagents: effects on the transport activity and transition from carrier to pore-like function. Biochim. Biophys. Acta 1611, 123-130.

Xu, Y., Mayor, J. A., Gremse, D., Wood, D. O. and Kaplan, R. S. (1995) High-yield bacterial expression, purification, and functional reconstitution of the tricarboxylate transport protein from rat liver mitochondria. Biochem. Biophys. Res. Commun. 207, 783-789.

Xu, Y., Kakhniashvili, D. A., Gremse, D. A., Wood, D. O., Mayor, J. A., Walters, D. E. and Kaplan, R. S. (2000) The yeast mitochondrial citrate transport protein. Probing the roles of cysteines, $\operatorname{Arg}(181)$, and $\operatorname{Arg}(189)$ in transporter function. $J$. Biol. Chem. 275, 7117-7124.

Wessel, D. and Flügge, U. I. (1984) A method for the quantitative recovery of protein in dilute solution in the presence of detergents and lipids. Anal. Biochem. 138, 141-143.

Zara, V. and Palmieri, F. (1988) Inhibition and labelling of the mitochondrial 2-oxoglutarate carrier by eosin-5-maleimide. FEBS Lett. 236, 493-496.

Zara, V., Iacobazzi, V., Siculella, L., Gnoni, G. V. and Palmieri, F. (1996) Purification and characterization of the tricarboxylate carrier from eel liver mitochondria. Biochem. Biophys. Res. Commun. 223, 508-513.

Zara, V., Palmieri, L., Franco, M. R., Perrone, M., Gnoni, G. V. and Palmieri, F. (1998) Kinetics of the reconstituted tricarboxylate carrier from eel liver mitochondria. J. Bioenerg. Biomembr. 30, 555-563.

Zara, V., Palmieri, L., Giudetti, A., Ferramosca, A., Capobianco, L. and Gnoni, G. V. (2000) The mitochondrial tricarboxylate carrier: unexpected increased activity in starved silver eels. Biochem. Biophys. Res. Commun. 276, 893-898.

Zara, V., Ferramosca, A. and Palmieri, L. (2003) Structural and functional properties of the mitochondrial metabolite carriers. Recent Res. Devel. Biochem. 4, 255-269. 